\title{
The Arteriovenous Loop: Engineering of Axially Vascularized Tissue
}

\author{
Annika Weigand $^{a}$ Raymund E. Horch ${ }^{a} \quad$ Anja M. Boos ${ }^{a} \quad$ Justus P. Beier ${ }^{b}$ \\ Andreas Arkudas ${ }^{a}$ \\ a Department of Plastic and Hand Surgery and Laboratory for Tissue Engineering and \\ Regenerative Medicine, University Hospital of Erlangen, Friedrich-Alexander-University \\ Erlangen-Nürnberg (FAU), Erlangen, Germany; ${ }^{b}$ Department of Plastic Surgery, Hand and \\ Burn Surgery, University Hospital RWTH Aachen, Aachen, Germany
}

\section{Keywords}

Tissue engineering · Arteriovenous loop · Animal models · Vascularization · Regenerative medicine

\begin{abstract}
Background: Most of the current treatment options for large-scale tissue defects represent a serious burden for the patients, are often not satisfying, and can be associated with significant side effects. Although major achievements have already been made in the field of tissue engineering, the clinical translation in case of extensive tissue defects is only in its early stages. The main challenge and reason for the failure of most tissue engineering approaches is the missing vascularization within large-scale transplants. Summary: The arteriovenous (AV) loop model is an in vivo tissue engineering strategy for generating axially vascularized tissues using the own body as a bioreactor. A superficial artery and vein are anastomosed to create an AV loop. This AV loop is placed into an implantation chamber for prevascularization of the chamber inside, e.g., a scaffold, cells, and growth factors. Subsequently, the generated tissue can be transplanted with its vascular axis into the defect site and anastomosed to the local vasculature. Since the blood supply of the growing tissue is based on the AV loop, it will be immediately perfused with blood in the recipient site leading to optimal healing conditions even in the case of poorly vascularized defects. Using this tissue engineering approach, a multitude of different axially vascularized tissues could be generated, such as bone, skeletal or
\end{abstract}

This paper is part of the special topic issue "Vascularization, Regeneration and Tissue Engineering." 
heart muscle, or lymphatic tissues. Upscaling from the small animal AV loop model into a preclinical large animal model could pave the way for the first successful attempt in clinical application. Key Messages: The AV loop model is a powerful tool for the generation of different axially vascularized replacement tissues. Due to minimal donor site morbidity and the possibility to generate patient-specific tissues variable in type and size, this in vivo tissue engineering approach can be considered as a promising alternative therapy to current treatment options of large-scale defects.

(C) 2018 S. Karger AG, Base

\section{The Technical Challenge: Reconstruction of Large-Scale Tissue Defects}

Tissue defects caused by orthopedic trauma or cancer can be associated with significant soft or hard tissue loss making surgical reconstruction necessary. Given the fact that the human life span is continuously increasing and that even far advanced age is no longer a real hindrance towards surgical interventions, a higher demand for tissue replacement is foreseeable [1]. Large-scale tissue defects often cause serious problems for patients and trauma surgeons. They are further associated with an enormous financial burden on healthcare systems and the economy. Even nowadays, with the assistance of state-of-the-art medical technology, restoring the function and appearance remains extremely challenging.

The gold standard therapy of such large defects still remains the transfer of autologous tissue. It provides optimal conditions in terms of osseointegration and osteoconductivity in case of bone defect reconstruction, due to the living cells and the cellular matrix within the transplant leading to rapid bone regrowth [2].

One of the biggest challenges for successful integration of the transplant and tissue regeneration involves the diffusion limitation which affects cells and therefore whole transplant survival [3]. While non-vascularized grafting can be chosen for small defects, large defects require the transplantation of vascularized grafts. To ensure immediate perfusion of the transplanted tissue after surgery, vascularized tissues such as local pedicled or free flaps are currently used for the treatment of soft and hard tissue defects [4, 5]. Depending on the site and size of the defects, as well as on properties of the flap, such as tissue composition and vascular supply [6], there are various autologous grafts that are currently used for transplantation, e.g., parts of the fibula, scapula, or the gastrocnemius muscle. However, the harvesting procedure creates an additional surgical site and can be associated with donor site morbidities such as loss or impairment of function, infection, swelling, contour deformities, and pain [7]. A further drawback of autologous transplants is the fixed shape of the grafts. For bone defects, grafts have to be adjusted by osteotomy to resemble the natural contouring which can be associated with the risk of devascularization since periosteal blood supply is often not macroscopically obvious [6]. With prefabrication techniques, it became possible to turn a random vascularized flap of desired shape, thickness, and texture into an axially vascularized flap [8]. Using this type of flap manipulation, autologous tissues, such as bone grafts, are implanted for several weeks into a highly vascularized area of the body, such as a fasciocutaneous pocket [9]. After adequate vascularization of the graft, it can be transplanted as a composite flap into the defect together with the surrounding tissue and its supporting vascular structures, leading to immediate vascular supply within the recipient area [9]. 
Weigand et al.: The Arteriovenous Loop

\section{Tissue Engineering Principle: Scaffolds, Cells, Growth Factors, and the Vascularization}

To overcome the aforementioned pitfalls of traditional therapies such as tissue harvesting, the concept of tissue engineering was developed some decades ago. This kind of technique to generate artificial tissues was originally based on three main pillars, also referred to as the "tissue engineering triad," namely a scaffold or a matrix for structural support, cells, and growth factors [10]. However, since the vascularization remains one of the key barriers for translation of tissue engineering-based strategies into clinics [11], it has become the main focus of many tissue engineering studies during recent years.

Vascularization strategies in tissue engineering can be divided into in vitro and in vivo approaches [12]. The in vitro generation of microvascular networks includes the fabrication of scaffolds with vascular-like internal channels, e.g., by bioprinting and further seeding vascular cells into these channels, such as endothelial cells or incorporation of angiogenic growth factors within the scaffold. On the other hand, in vivo strategies are using the body itself as a bioreactor. In a two-step process, prevascularization can be achieved by the implantation of scaffolds into a highly vascularized area such as the subcutaneous tissue. These constructs will be "extrinsically" vascularized by surrounding vessels [13]. However, the second step of this procedure - the transplantation into the defect site - goes along with a sudden interruption of the blood supply. For subsequent reconnection to the blood system, vessels have to anastomose immediately with the local vasculature in the defect area. If this process takes too long and cells are more than 100-200 $\mu \mathrm{m}$ away from the nearest capillary they cannot be adequately supplied with nutrients and oxygen [14]. Based on the principles of the prefabrication strategy of flaps, attempts have been made to induce an "intrinsic" or "axial" vascularization within the scaffolds, allowing microvascular transplantation after prevascularization [13]. Either existing blood vessels of the body or a microsurgically created vascular loop between an artery and vein can serve as the vascular axis, which itself will become the main supporting blood vessel for the construct. It can be re-anastomosed to the local vasculature at the recipient site after transplantation, allowing immediate blood supply and integration of the generated tissue. Using this approach, Warnke et al. [15] could successfully transplant an in vivo tissue-engineered bone construct into a mandibular defect of a patient after vascularization within the latissimus dorsi muscle.

\section{The Arteriovenous Loop Model: Method and Mechanism behind the Axial Vascularization}

The prevascularization by an arteriovenous (AV) shunt or the so-called AV loop strategy was originally developed by Erol and Spira [16] in a rat model for skin engineering. Only some years later their hypothesis that the AV loop "could possibly vascularize a digital replantation with severely damaged vessels and provide enough additional vascularity to facilitate the take of bone grafts and tissue composites" became true [16]. Nearly 40 years later, a range of publications around the AV loop model can be found in the literature, including the generation of different tissue types at different locations, the upscaling from small to large animals, and recently the first steps towards clinical translation and further even modification of the AV loop model for setting up a novel disease model.

The experimental AV loop is mainly described in the rat. By using an operating microscope, the femoral vessels in the inguinal region of the rat are microsurgically prepared. The femoral artery and vein are anastomosed with an interpositional venous graft (IVG) of about $1.5 \mathrm{~cm}$ from the contralateral side by $10-0$ or $11-0$ sutures (Fig. 1 ). The IVG is necessary to achieve 

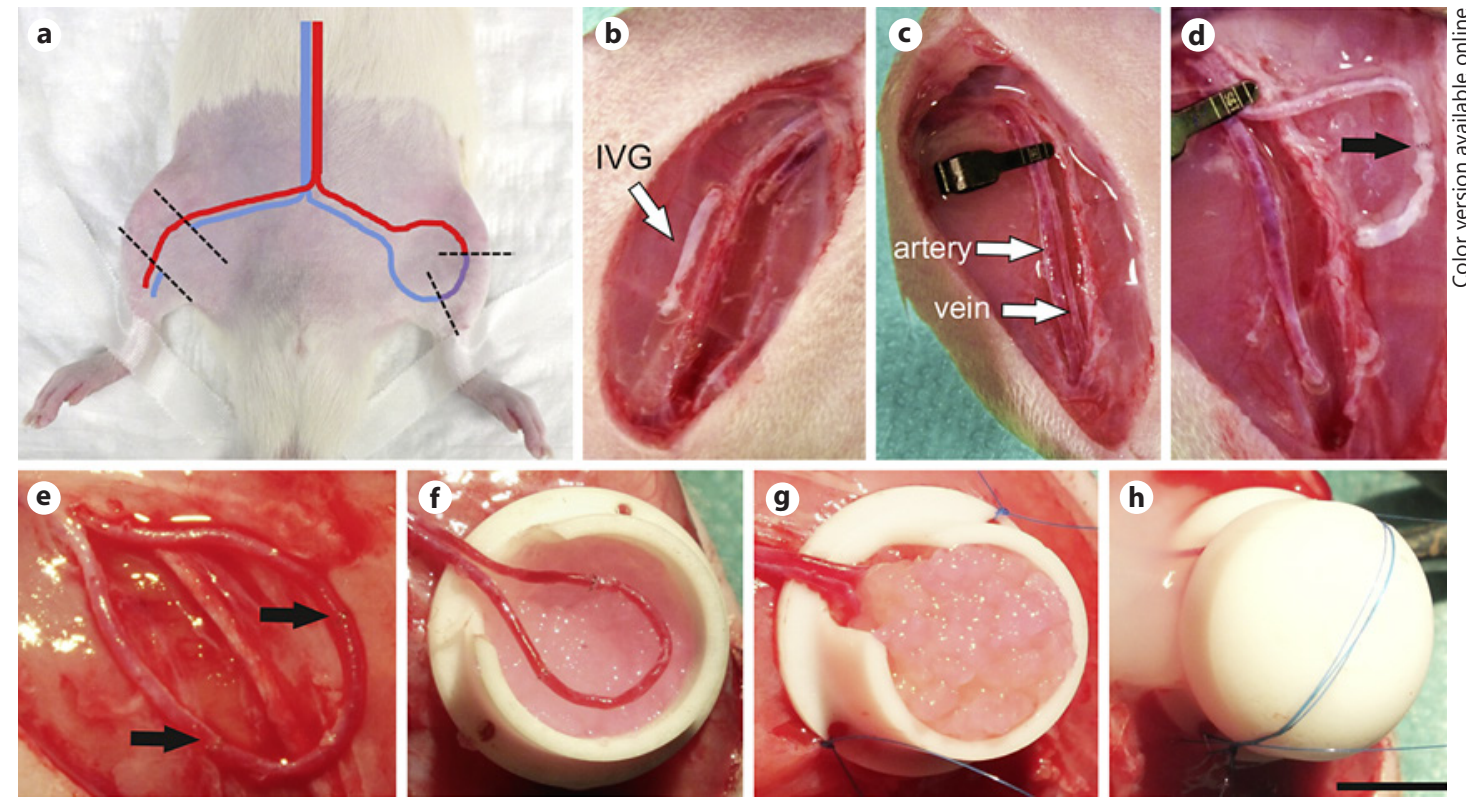

Fig. 1. Arteriovenous loop operation in the rat model. a Scheme of the AV loop principle in the rat model. The femoral artery and vein are anastomosed into an AV loop by interposing a venous graft from the contralateral side of the animal. b Harvesting of the IVG from the right side of the animal. c Microsurgical preparation of the femoral vessels. $\mathbf{d}$ Anastomosis of the IVG in between the vein and artery. e Generation of the AV loop after the second anastomosis (arrows). $\mathbf{f}$ AV loop in a polycarbonate chamber prefilled with a hydrogel. $\mathbf{g}$ Complete filling of the chamber with a hydrogel and fixation of the chamber in the groin of the rat. $\mathbf{h}$ Chamber with closed lid, the entrance and AV loop are embedded in fibrin. Scale bar, $5 \mathrm{~mm}$.

sufficient length of the loop for tension-free anastomosis. Over time, the process of arterialization of the venous graft could be shown by a reduction in vessel diameter after 7 days [17]. Polykandriotis et al. [17] could demonstrate that in addition to the venous part, the IVG serves as an inductor of early angiogenesis. These findings corroborate the results of Schmidt et al. [18] and Arkudas et al. [19] showing that early vessel sprouting mainly originates from the IVG. They further conclude that the hemodynamic changes to a more turbulent flow lead to connexin 43 expression within the IVG and could perhaps be a trigger for neoangiogenesis [18].

The AV loop can be placed in a cylindrical plastic (e.g., polycarbonate) chamber together with a matrix as described by Hofer et al. [20] or Tanaka et al. [21], finally closed, and implanted for several weeks for vascularization. Apart from the entrance for the loop vessels, the chamber is completely sealed from the environment enabling solely axial vascularization by the AV loop. This further leads to hypoxia within the chamber, which itself is an effective stimulus for neoangiogenesis. By using pimonidazole hydrochloride injection, Lokmic et al. [22] could demonstrate that hypoxia and proliferation of cells within the AV loop chamber peaked at 7 days. Likewise, Yuan et al. [23] reported that hypoxia distribution shown by HIF- $1 \alpha$ staining within the AV loop construct correlates with the degree of vascularization. The application of a prolyl hydroxylase inhibitor led to upregulation of HIF- $1 \alpha$ and subsequent increase in vessel growth, claiming that HIF-1 $\alpha$ is a key element for vessel outgrowth from the loop [24]. Besides, a Nox2-containing nicotinamide adenine dinucleotide phosphate (NADPH) oxidase is known to play a major role in hypoxia-driven angiogenesis. Recently, it could be confirmed that it is involved in the process of neoangiogenesis within the AV loop chamber and that specific inhibitors such as apocynin or gp91ds-tat peptide lead to an inhibition of further vessel growth [25]. 


\section{Shape Adjustment of the Implantation Chamber}

The implantation chamber can be adjusted in size and shape to match the requirements needed for the tissue-engineered construct. Cassell et al. [26] implanted different matrices in the AV loop chamber and observed shrinkage of the constructs after reaching maximum size in all groups, probably due to capsule formation in response to the walls of the hydrophobic polycarbonate chamber. Hofer et al. [20] could show that an increase of the chamber size from 0.45 to $1.9 \mathrm{~mL}$ while using the same-size vessel loop could generate larger quantities of tissue. The modification of the chamber from a closed to a perforated one has been described by Dolderer et al. [27] and Arkudas et al. [28]. Dolderer et al. [27] implanted a perforated polycarbonate chamber for 4 weeks in the rat model and measured a higher amount of tissue growth within perforated compared to closed chambers. Most probably this is due to a faster ingrowth of tissue and cells through the perforations [27]. This finding supports the study of Arkudas et al. [28], in which perforated titanium chambers were found to be superior for tissue engineering compared to closed ones. Angiogenesis within perforated chambers was enhanced by the additional extrinsic vascular pathway. Notably, the AV loop vessels showed connections with the extrinsic vasculature even after 2 weeks of implantation, making the microvascular transplantation of the whole construct possible. Likewise, we implanted perforated titanium chambers in the sheep AV loop model and could demonstrate a higher degree of tissue ingrowth and remodeling of the bone replacement material compared to closed chambers. This can lead to a significant reduction in prevascularization time and earlier transplantation of the generated tissue [29].

In order to take the next step of transplantation of the axial vascularized tissue into a defect, Arkudas et al. [30] modified the perforated chamber allowing integration into a femoral defect site after vascularization. For this purpose, two wings were added to the chamber for later fixation with screws on the femur. Besides, two holes were added to each side of the defect chamber to ensure direct contact of the scaffold with the femoral stumps.

\section{The AV Loop as a Tool to Generate a Multitude of Different Tissues}

Since the first and successful attempt to generate axially vascularized skin by Erol and Spira [16], a high variety of tissue types have been grown in the AV loop model. With the discovery of the possibility to vascularize different kinds of soft and hard matrices, researchers began to combine them with specific cell types, growth factors, or even nerves steering the differentiation and growth of the construct inside the camber towards the desired tissue type.

The majority of experimental AV loop studies in the field of tissue engineering have focused on the generation of bone tissue. A lot of different bone substitutes were implanted, including stable [31] or non-stable scaffolds [30, 32-34] composed of $60 \%$ hydroxyapatite (HA) and $40 \% \beta$-tricalcium phosphate ( $\beta$-TCP) [30-34] or $100 \% \beta$-TCP [35], natural coral scaffolds [36], processed bovine cancellous bone [37], or nanocrystalline HA in a silica gel matrix $[29,38]$. To functionalize the scaffolds and to support bone formation, cells can be combined with the replacement material, e.g., non-differentiated bone-marrow derived mesenchymal stem cells (MSCs) [30, 32, 34, 39] or fully differentiated osteogenic cells such as osteoblasts [40]. MSCs are often used in tissue engineering studies due to their multipotent differentiation capacity, their self-renewal and secretion of various growth factors that stimulate angiogenesis, recruitment of other cells, and further regulate the immune response. Their differentiation can be controlled by the addition of specific growth factors. For the longterm delivery of growth factors they can also be directly incorporated into the scaffold to ensure a continuous release over several weeks [39]. In bone tissue engineering, bone 
morphogenetic protein-2 (BMP-2) significantly stimulates bone formation [31, 38] and is often combined with MSCs [30, 32, 34]. Buehrer et al. [34] recently showed that the combination of BMP-2 and MSCs leads to significantly higher bone formation in the AV loop compared to the application of only BMP-2 or only MSCs.

Besides bone, there are studies focusing on the generation of axially vascularized skeletal or heart muscle tissues. Messina et al. [41] transplanted pieces of skeletal muscle tissue from the rat and human into AV loop chambers and observed the formation of both adipogenic and striated skeletal muscle fibers. Likewise, the implantation of Matrigel ${ }^{\circledR}$ and myoblasts also induced desmin-positive muscle fibers, however without the formation of adipogenic tissue. By implanting the proximal femoral and epigastric nerves for innervation of the muscle, the amount of muscle tissue increased at 6 weeks of implantation [41]. Beier and colleagues [42] modified the AV loop model for muscle tissue engineering into the so-called EPI loop model. The superficial epigastric vein and the saphenous artery are anastomosed via an IVG from the superficial inferior epigastric vein allowing the additional implantation of the obturator nerve for motoric innervation. To ensure survival of the implanted cells and the nerve within the chamber, a collagen-fibrin matrix was prevascularized for 2 weeks before adding MSC, myoblasts, and the proximal end of the obturator nerve into the chamber. After 8 weeks, myogenic differentiation of the vascularized tissue inside the chamber could be shown [42]. Generation of skeletal muscle tissue and differentiation can be supported by providing an optimal 3D structure for guiding the tissue growth and allowing matrix deposition and cellular interactions. By means of electrospinning of parallel aligned fibers and further cocultivation of myoblasts and MSCs on this scaffold, Beier and colleagues [43,44] recently created favorable conditions for further scaffold-based muscle tissue engineering studies in the AV loop model (Fig. 2).

Heart muscle tissue was successfully generated by Tee et al. [45, 46]. Neonatal cardiomyocytes were implanted into the AV loop in Matrige ${ }^{\circledR}$, resulting in the growth of a contractile muscle that stained positive for typical cardiac muscle markers. As proof of concept, vascularized transplantation of the generated tissue to the heart of a recipient animal was performed. After 2 weeks of transplantation the flaps stayed vital, showing contractions, and the cardiomyocytes maintained markers of a mature phenotype [45].

There have been a few more studies focusing on the development of axially vascularized soft tissue types. Schmidt et al. [47] published the generation of axially vascularized soft tissue as therapy for large wounds by using collagen-elastin and collagen-glycosaminoglycan scaffolds. Comparable to Tee et al. $[45,46]$, they could show the successful transplantation in a rat model, thereby increasing the probability of the translation into clinics in near future. Brown et al. [48] worked on the establishment of an axially vascularized pancreatic organoid for therapy of diabetes and as an alternative to challenging transplantations of insulinproducing tissues. They were able to grow functional pancreatic isle tissue within a Matrigel ${ }^{\circledR}$ matrix. Implanted cells remained functional and hormone producing after an implantation time of 3 weeks. Besides using this approach as a possible new treatment option in case of the dysfunction of pancreatic islet cells, it also opens up new ways for studying the transplantation of pancreatic and further tissues [48]. Fiegel et al. [49] attempted to generate an organ with similar complexity, the liver, within the AV loop model. Fetal liver cells were transplanted within a fibrin-matrix into the rat model. After 2 weeks, the matrix was highly vascularized and the fetal liver cells could be redetected providing the way for further studies concentrating on the generation of functional and axially vascularized hepatic tissue [49].

Although tissue engineering of functional lymphatic vascular structures and lymphatic tissue is still at a very early stage, it has been increasingly gaining attention due to the high clinical demand. People suffering from primary or secondary lymphedema often cannot be treated adequately. Non-surgical therapies are time-consuming, associated with loss of quality 

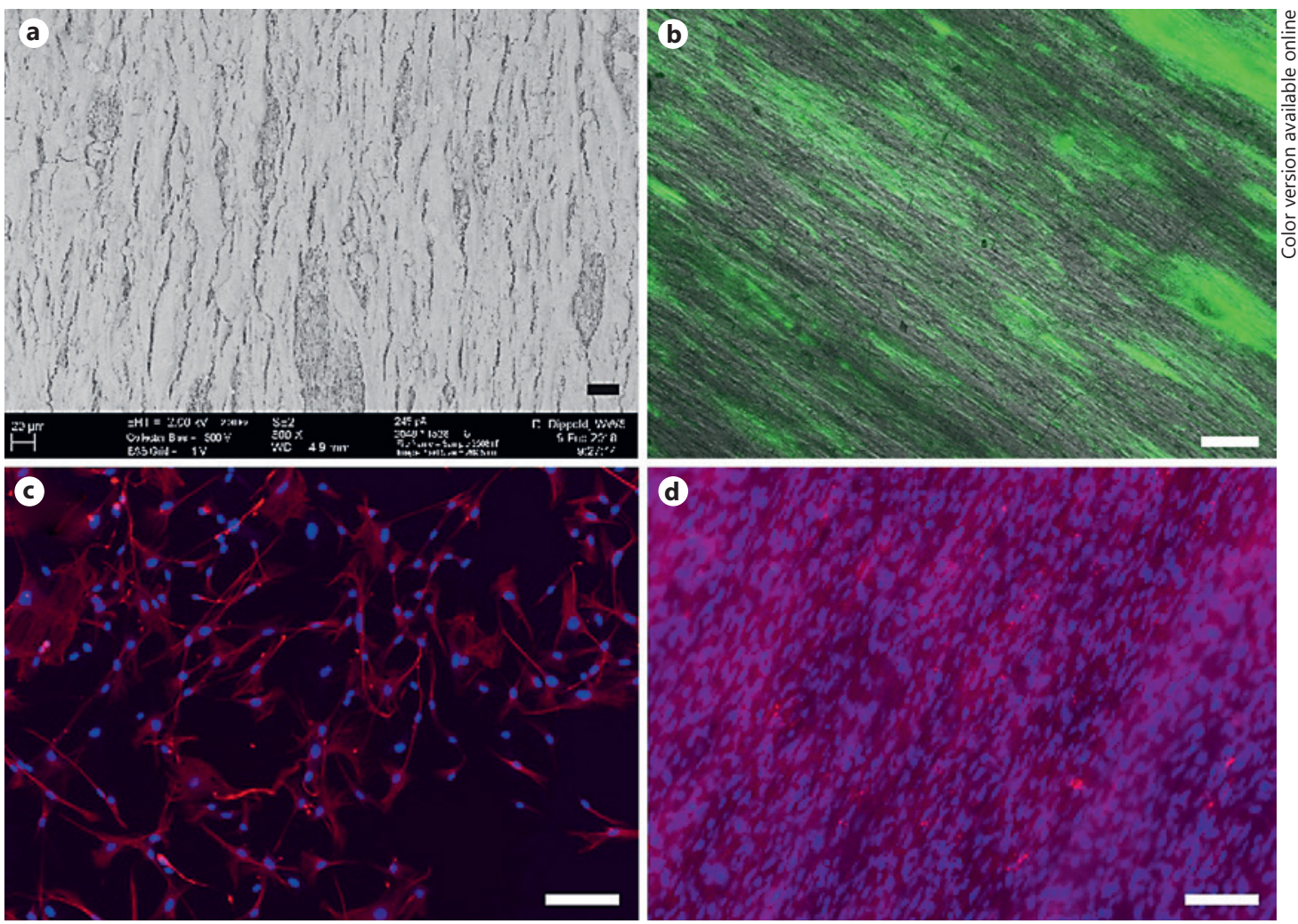

Fig. 2. Tissue engineering of muscle. a SEM image of an electrospun parallel-orientated PCL-collagen-I nanofiber scaffold after seeding the myoblast mouse cell line $\mathrm{C} 2 \mathrm{C} 12$. Scale bar, $20 \mu \mathrm{m}$. b Coculture of rat myoblasts with GFP-labeled bone marrow-derived MSC on an electrospun parallel-orientated PCL-collagen-I nanofiber scaffold. Scale bar, $200 \mu \mathrm{m}$. c Desmin staining (red) of primary rat myoblasts, isolated with the preplate method, counterstaining with DAPI (blue). Scale bar, $100 \mu \mathrm{m}$. d Coculture of rat myoblasts and adipose-derived stem cells on an electrospun parallel-orientated PCL-collagen-I nanofiber scaffold, MHC staining (red), counterstaining with DAPI. Scale bar, $100 \mu \mathrm{m}$.

of life, and cannot stop the disease or achieve a complete cure. Surgical methods are associated with donor site morbidities and often lead to poor outcomes [50]. To bypass the problem of harvesting own tissue, such as a lymph nodes, a range of tissue engineering approaches have been developed during recent years, such as coculturing of lymphatic endothelial cells with stem cells, developing hydrogels, and using growth factors or stimulation with interstitial flow [51]. Robering et al. [52] are currently working on the establishment of an autonomous axially vascularized lymphatic tissue using the rat AV loop model. Stem cells could significantly stimulate angiogenic properties of lymphatic endothelial cells in a range of angiogenesis assays. The first attempts of co-injection of lymphatic endothelial cells and MSCs led to the formation of LYVE-1 and podoplanin-positive lymphatic vascular structures in the AV loop model (Fig. 3).

\section{Animal Models}

Even though small animal models have advantages, such as availability, easy handling and breeding, and lower costs, larger animals are of utmost importance for later clinical translation. Besides in the rat, the AV loop is described in animals such as the rabbit, dog, goat, and sheep. 


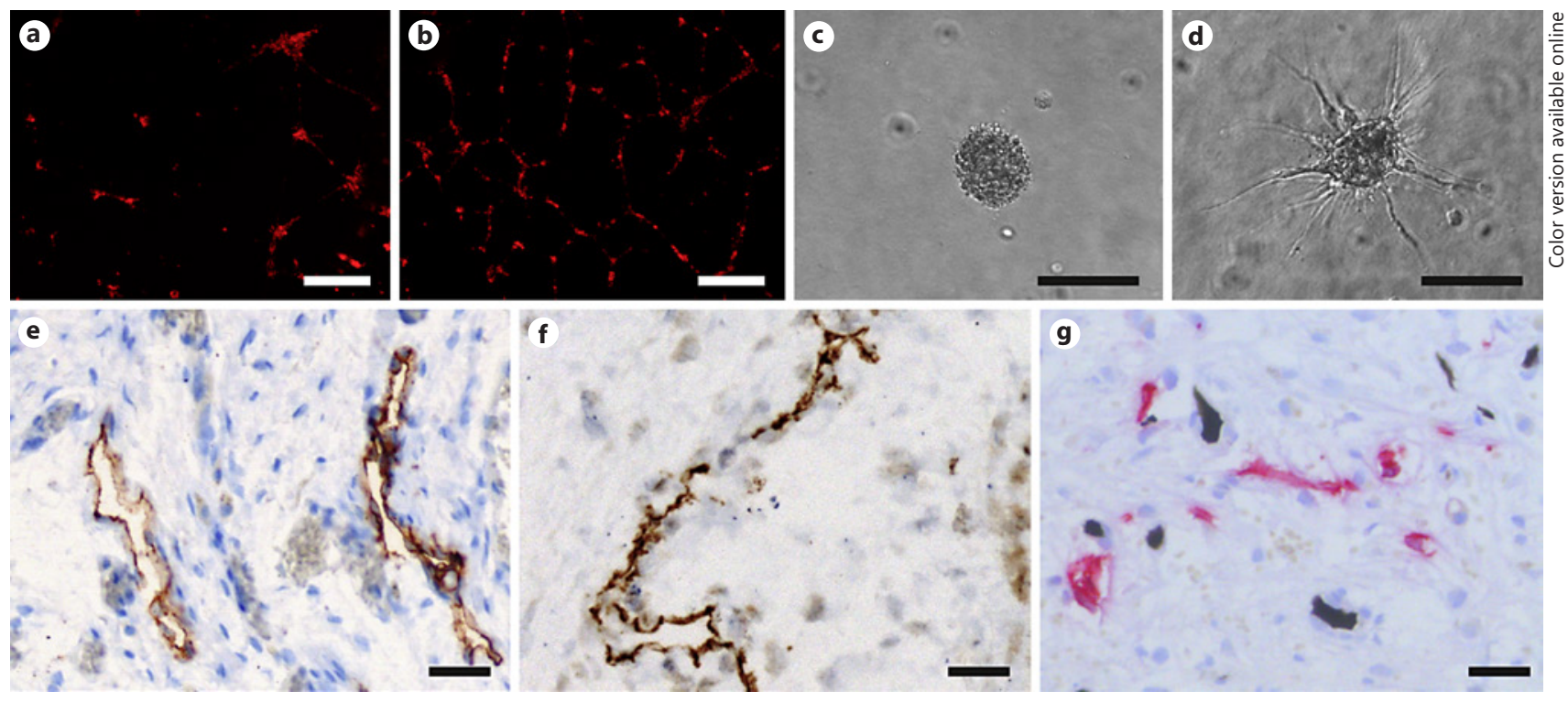

Fig. 3. Tissue engineering of lymphatic structures. Tube formation assay of lymphatic endothelial cells (LECs) in control medium (a) and in conditioned medium of CM-DiI-labeled (red) MSCs (b). Scale bars, 200 $\mu \mathrm{m}(\mathbf{a}, \mathbf{b})$. Sprouting assay of LECs in control medium (c) and in conditioned medium of MSCs (d) after $24 \mathrm{~h}$. Scale bars, $100 \mu \mathrm{m}$ (c, d). e-g Implantation of human LECs and MSCs into the rat AV loop model for the generation of an axially vascularized lymphatic tissue. Rat podoplanin-positive (e), human lyve 1-positive (f), and human CD31-positive (g) vessels could be detected after an implantation time of $2(\mathbf{g})$ and 4 weeks $(\mathbf{e}$, f). Scale bars, $50 \mu \mathrm{m}(\mathbf{e}-\mathbf{g})$.

For the generation of larger engineered bone tissue, Eweida et al. [53] transferred the AV loop to the rabbit model. Chambers with a diameter of $18 \mathrm{~mm}$ and a height of $0.8 \mathrm{~mm}$ were implanted. Although not using a venous graft as commonly performed in the rat model, the AV loop could be generated without tension by anastomosing the saphenous vessels. However, 2 out of 6 animals had to be excluded due to vascular thrombosis. Dong et al. [36] also used the rabbit model, but took a different approach to vascularize a construct of $6 \times 8 \times 10 \mathrm{~mm}$. Instead of using the femoral vascular bundle like for the rat model or the saphenous vascular bundle as described by Eweida et al. [53], the popliteal artery was anastomosed with the femoral vein. The length of the popliteal artery enabled them to omit the vascular graft and, hence, reduce the risk of vascular thrombosis. Only 2 out of 64 rabbits had to be excluded due to thrombosis.

Wu et al. [35] used the dog for generation of an axially vascularized bony construct. The saphenous artery and vein served as loop vessels and a $\beta$-TCP matrix and bone marrow were implanted without using a chamber. After 6 months, a high degree of bone formation and vessel sprouting was visible, however, the constructs, cylinders of $1.2 \times 1 \mathrm{~cm}$, were quite small.

Eweida et al. [31] implanted a $\beta$-TCP/HA scaffold more than twice as big $(2 \times 3 \mathrm{~cm})$ together with platelet-rich plasma and BMP-2 into a mandibular defect of goats using the facial vessels. There was a significant higher site-specific bone formation in the AV loop groups compared to non-vascularized scaffolds. Beier et al. [54] developed the AV loop large animal model for long bone defects in the sheep using a chamber measuring $2.8 \times 1.8 \mathrm{~cm}$ with a height of $1.8 \mathrm{~cm}$ and a total volume of $16 \mathrm{~cm}^{3}$. Comparable to Wu et al. [35], the saphenous artery and vein in the groin of the animals served as AV loop vessels. This model was further adapted and applied in a range of bone tissue engineering studies with different chamber 

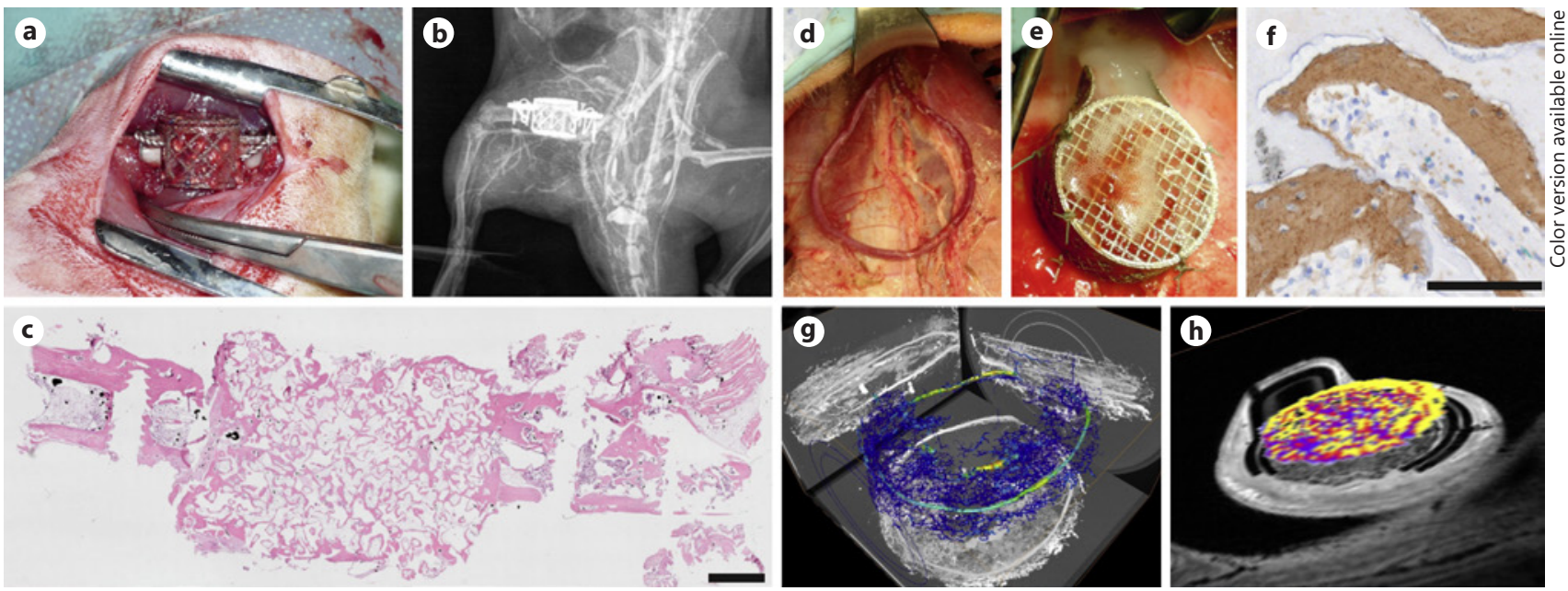

Fig. 4. Bone tissue engineering in the small and large animal model. a-c Critical size femur defect model in the rat. a Pedicled transplantation of a prevascularized bone scaffold within a perforated titanium chamber for combined extrinsic-intrinsic vascularization into a critical size femur defect. b X-ray of the implanted chamber. c HE staining of the femur with the transplanted bone construct consisting of HA/ $\beta$-TCP, MSC, and BMP-2 after 12 weeks of implantation. Scale bar, $2 \mathrm{~mm}$. $\mathbf{d}-\mathbf{h}$ Bone tissue engineering in the sheep (large animal). d An AV loop is generated by anastomosing the saphenous artery and vein. e For combined extrinsicintrinsic vascularization the AV loop and a bone substitute are implanted within a perforated titanium chamber. $\mathbf{f}$ Collagen type I-positive tissue (brown) after implantation in the sheep AV loop model. Scale bar, 100 $\mu \mathrm{m} . \mathbf{g}, \mathbf{h}$ With 3D imaging vascularization and perfusion of the constructs can be visualized using micro-CT (g) and oxygen measurement (h).

architectures, bone substitutes, cells, and growth factors [29, 32]. Since it was possible to axially vascularize constructs with a "clinically relevant dimension," this model may be suitable for further preclinical research [54].

\section{From Basic Research to Clinical Application}

Besides upscaling from small to large animals, a fundamental requirement for translation from bench to beside is the approval of the microvascular transplantation of the AV loopengineered tissues. Schmidt et al. [55] have postulated the successful transfer of generated soft tissue on complex scapular defects, including skin, fascia, and muscle, in a rat model. Further studies with large animals are recommended to provide support for introducing this approach as an alternative to conventional autologous free flaps. Tee et al. $[45,46]$ generated contractile cardiac muscle tissue within an axially vascularized chamber in the rat model and transplanted it based on the femoral-iliac-aortic vascular axis to the heart muscle of recipient rats. Arkudas et al. [30] could show in a range of studies that they are able to generate axially vascularized bone tissue in a rat model. They recently postulated the successful pedicled transplantation of an AV loop construct consisting of a $\beta$-TCP/HA, MSC, and recombinant BMP-2 into a critically sized rat femoral defect of $1 \mathrm{~cm}$. With 3D imaging techniques, histology and gene expression studies enhanced vascularization and bone bridging in groups with the AV loop compared to non-vascularized scaffolds, which could be demonstrated after 12 weeks (Fig. $4 \mathrm{a}-\mathrm{C}$ ). Based on the results of our previous studies with the AV loop sheep model (Fig. 4d-h), the step from the small to the large animal is currently being performed in our laboratory. For this, a scaffold with a volume of about $8 \mathrm{~cm}^{3}$ is prevascularized by an AV loop 
within a custom-made titanium chamber, allowing intrinsic-extrinsic vascularization. After 4 weeks, a tibia defect with a size of $3 \mathrm{~cm}$ is generated and the prevascularized construct transplanted and anastomosed to vessels in the recipient area.

Horch et al. [56] recently published the successful application of in situ tissue engineering with the AV loop technique in 2 patients with large bone defects of the tibia $(8 \times 2.5 \mathrm{~cm})$ and of the radius $(3 \times 4 \mathrm{~cm})$, respectively. An AV loop was created directly at the defect site and defects either filled with autologous bone from the iliac crest or with the bone substitute material $\beta$-TCP/HA in combination with bone marrow aspirate and fibrin. In the long-term follow-up, several years after surgery, stable bony bridging of the defects could be proven. The 2 patients regained full mobility and were pain free.

\section{Challenges}

According to Zhan et al. [57] the critical factors for a successful AV loop surgery are "surgical technical, blood flow, thrombosis and spasm." A retrospective analysis of more than $600 \mathrm{AV}$ loop surgeries showed a significant correlation of experience and practice of microsurgeons and the thrombosis rate and time of surgery [58]. For the reduction of vessel thrombosis, a range of different antiplatelet and/or anticoagulant drugs can be applied in the postoperative phase such as heparin, sodium-enoxaparin, or clopidogrel [18, 24, 59]. To reduce bending of the loop vessels and therefore the risk of thromboses, Zhan et al. [57] suggest using the epigastric branches as tethers to hold the AV loop in position - or as an alternative, nylon stitches from the wall of the AV loop vessel to the surrounding tissue. Dong et al. [36] tried to reduce thrombosis by reducing the number of anastomoses. Instead of using the IVG, they anastomosed the popliteal artery with the femoral vein and therefore could generate an AV loop that was long enough even without the IVG. Likewise, in larger animals, there is no need for an IVG between the artery and vein $[29,31,35,54]$. However, it has to be kept in mind that the IVG has been considered by a range of researchers to be a decisive element in the early phase of vascular sprouting in the AV loop model [17-19].

Another strategy to induce axial vascularization with a concurrent reduction of the risk of a vascular thrombosis is the so-called vascular bundle model [57]. It can be further divided into the "flow through" or "distal ligation" model [60]. In most studies using the AV bundle, the vessels are ligated and vascular sprouts originate from the pedicle ends. Surgery time can be reduced and microsurgeons do not need a lot of experience. However, in many studies the vascularization by the AV loop was found to be superior compared to the AV bundle [35, 36, 60,61 ]. According to $\mathrm{Wu}$ et al. [35] the vascular bundle model achieved a lower degree of vascularization but simultaneously a better balance between bone formation and scaffold absorption and a higher success rate. In a skin tissue engineering study, the AV loop showed a significantly higher potential for tissue growth and vessel sprouting. However, the AV bundle distal ligation also seems to be an effective model for the generation of axially vascularized skin flaps [60]. Dong et al. [36] also observed a higher vascular sprouting from the AV loop compared to the AV bundle. They clearly recommend the AV loop for bone tissue engineering.

\section{Potential Applications of the AV Loop Model Beyond Tissue Engineering}

Originally conceived and developed for tissue engineering, a range of ideas have arisen about using the AV loop for other purposes. Since the inner of the chamber is completely isolated from the living organism - except the connection by the AV loop vessels - there is 
the unique opportunity to analyze vasculogenic and angiogenic processes in vivo without interfering influences from the surrounding tissue [62]. The effect of different substances, such as specific growth factors or cells on vascularization, can intensively be examined by using in vivo 3D imaging methods, such as angio-CT, MRI, or postmortem with micro-CT, corrosion cast techniques, or histology. For example, the effect of different concentrations of the angiogenic growth factors VEGF and bFGF on vascular sprouting was analyzed, indicating a dose-dependent effect up to a concentration of $100 \mathrm{ng} / \mathrm{mL}$ [19]. In view of generating AV loop-engineered tissues for patients with tissue defects due to cancer and subsequent radiotherapy, Schmidt et al. [63] analyzed the effect of ionizing radiation on vascular sprouting of the IVG. As previously described, the vascular graft may be a trigger of neoangiogenesis in the AV loop constructs and would therefore be of utmost importance for the vascularization process. Vascular sprouting was significantly reduced after a single dose of 2 Gy - a noteworthy finding that should be considered in the case of treatment of patients after radiotherapy with the AV loop strategy [63]. Further potential applications may be seen in a combination of modern biofabrication techniques together with prevascularized custom-made bioprinted scaffolds, which allow a distinct deposition of cells into a matrix [64].

Besides using the AV loop for tissue engineering or as model for angiogenesis, it was recently suggested to be used as a platform for cancer research $[65,66]$. Most cancer models are based on the subcutaneous or intravenous injection of tumor cells. Developing tumors, either under the skin or at organ-specific sites, are always influenced by the surrounding tissue. Based on the experiences with the AV loop as a tissue engineering model, it is conceivable that different tumor types can be grown within the isolation chamber. The tissue growth and, furthermore, the development of tumor vessels in such a controlled but manipulatable environment would allow the specific analysis of tumor progression and angiogenesis. Moreover, the effect on tumor and vessel growth of specific cancer drugs could be tested within this model. However, it also has to be recognized that the complexity of the surgery demands a high level of microsurgical expertise and therefore should not be seen as a screening tool, but as a model to answer specific questions [17].

\section{Acknowledgments}

We would like to thank the following institutions for supporting our AV loop research: Staedtler Stiftung; Dr. Fritz Erler Fonds; Else Kröner Fesenius Stiftung; Baxter Healthcare GmbH; German Research Foundation (DFG) within the collaborative research center TRR225 (subproject C03/04); IZKF/ELAN/EFI/Office for Gender and Diversity/Marohn-Stiftung/Forschungsstiftung Medizin, Friedrich-Alexander University of Erlangen-Nürnberg (FAU); the AO Foundation (Grant S-10-36A); Manfred Roth Stiftung; Xue Hong and Hans Georg Geis Foundation.

We would like to thank Dr. Amelie Lipp, Dr. Aijia Cai, Prof. Dr. Tobias Bäuerle, Jutta Jordan, Marc Schwarz, Rafael Schmid, Dr. Ran An, Jan Robering, Majida Al-Abboodi, Prof. Dr. Andreas Hess for their contribution to the animal experiments and providing us with pictures. We would like to thank Stefan Fleischer, Marina Milde, Katrin Köhn, and Ilse Arnold-Herberth for their excellent technical support.

\section{Disclosure Statement}

The authors declare that they have no potential conflicts of interest. 
Weigand et al.: The Arteriovenous Loop

\section{References}

1 Ludolph I, Lehnhardt M, Arkudas A, et al: Plastic reconstructive microsurgery in the elderly patient - consensus statement of the German Speaking Working Group for Microsurgery of the Peripheral Nerves and Vessels (in German). Handchir Mikrochir Plast Chir 2018;50:118-125.

2 Huang RL, Kobayashi E, Liu K and Li Q: Bone graft prefabrication following the in vivo bioreactor principle. E Bio Med 2016;12:43-54.

3 McMurtrey RJ: Analytic models of oxygen and nutrient diffusion, metabolism dynamics, and architecture optimization in three-dimensional tissue constructs with applications and insights in cerebral organoids. Tissue Eng Part C Methods 2016;22:221-249.

4 Zheng L, Lv X, Zhang J, Zhang Y, Cai Z, Liu S: Deep circumflex iliac artery perforator flap with iliac crest for oromandibular reconstruction. J Craniomaxillofac Surg 2018;46:1263-1267.

5 Schreiber M, Dragu A: Free temporal fascia flap to cover soft tissue defects of the foot: a case report. GMS Interdiscip Plast Reconstr Surg DGPW 2015;4:Doc01.

6 Ozkan 0: Single osteotomized iliac crest free flap in anterior mandible reconstruction. Microsurgery 2006;26: 93-99.

7 Lakhiani C, DeFazio MV, Han K, Falola R, Evans K: Donor-site morbidity following free tissue harvest from the thigh: a systematic review and pooled analysis of complications. J Reconstr Microsurg 2016;32:342-357.

8 Wang W, Zhao M, Tang Y, et al: Long-term follow-up of flap prefabrication in facial reconstruction. Ann Plast Surg 2017;79:17-23.

9 Douglas AJ, Kyzas PA: A new autologous block-bone prefabricated flap concept based on the supraclavicular artery island flap (SCAIF) for reconstruction of a neo-mandibular osteoradionecrosis (ORN) defect, IDEAL Stage 1 report. JPRAS Open 2017;12:19-24.

10 O'brien FJ: Biomaterials and scaffolds for tissue engineering. Mater Today 2011;14:88-95.

11 Baranski JD, Chaturvedi RR, Stevens KR, et al: Geometric control of vascular networks to enhance engineered tissue integration and function. Proc Natl Acad Sci USA 2013;110:7586-7591.

12 Auger FA, Gibot L, Lacroix D: The pivotal role of vascularization in tissue engineering. Ann Rev Biomed Eng 2013;15:177-200.

13 Lokmic Z, Mitchell GM: Engineering the microcirculation. Tissue Eng Part B Rev 2008;14:87-103.

14 Rouwkema J, Rivron NC and van Blitterswijk CA: Vascularization in tissue engineering. Trends Biotechnol 2008;26:434-441.

15 Warnke PH, Springer IN, Wiltfang J, et al: Growth and transplantation of a custom vascularised bone graft in a man. Lancet 2004;364:766-770.

16 Erol 00, Spira M: New capillary bed formation with a surgically constructed arteriovenous fistula. Plast Reconstr Surg 1980;66:109-115.

17 Polykandriotis E, Tjiawi J, Euler S, et al: The venous graft as an effector of early angiogenesis in a fibrin matrix. Microvasc Res 2008;75:25-33.

18 Schmidt VJ, Hilgert JG, Covi JM, et al: High flow conditions increase connexin43 expression in a rat arteriovenous and angioinductive loop model. PLoS One 2013;8:e78782.

19 Arkudas A, Pryymachuk G, Hoereth T, et al: Dose-finding study of fibrin gel-immobilized vascular endothelial growth factor 165 and basic fibroblast growth factor in the arteriovenous loop rat model. Tissue Eng Part A 2009;15:2501-2511.

20 Hofer SO, Knight KM, Cooper-White JJ, et al: Increasing the volume of vascularized tissue formation in engineered constructs: an experimental study in rats. Plast Reconstr Surg 2003;111:1186-1194.

21 Tanaka Y, Tsutsumi A, Crowe DM, Tajima S, Morrison WA: Generation of an autologous tissue (matrix) flap by combining an arteriovenous shunt loop with artificial skin in rats: preliminary report. Br J Plast Surg 2000;53: 51-57.

22 Lokmic Z, Stillaert F, Morrison WA, Thompson EW, Mitchell GM: An arteriovenous loop in a protected space generates a permanent, highly vascular, tissue-engineered construct. FASEB J 2007;21:511-522.

23 Yuan Q, Arkudas A, Horch RE, et al: Vascularization of the arteriovenous loop in a rat isolation chamber model - quantification of hypoxia and evaluation of its effects. Tissue Eng Part A 2018;24:719-728.

24 Yuan Q, Bleiziffer O, Boos AM, et al: PHDs inhibitor DMOG promotes the vascularization process in the AV loop by HIF-1a up-regulation and the preliminary discussion on its kinetics in rat. BMC Biotechnol 2014;14:112.

25 Jiang F, Zhang G, Hashimoto I, et al: Neovascularization in an arterio-venous loop-containing tissue engineering chamber: role of NADPH oxidase. J Cell Mol Med 2008;12:2062-2072.

26 Cassell OC, Morrison WA, Messina A, et al: The influence of extracellular matrix on the generation of vascularized, engineered, transplantable tissue. Ann NY Acad Sci 2001;944:429-442.

27 Dolderer JH, Kehrer A, Schiller SM, et al: De-novo generation of vascularized tissue using different configurations of vascular pedicles in perforated and closed chambers (in German). Wien Med Wochenschr 2010;160: 139-146.

28 Arkudas A, Pryymachuk G, Beier JP, et al: Combination of extrinsic and intrinsic pathways significantly accelerates axial vascularization of bioartificial tissues. Plast Reconstr Surg 2012;129:55e-65e.

29 Weigand A, Beier JP, Hess A, et al: Acceleration of vascularized bone tissue-engineered constructs in a large animal model combining intrinsic and extrinsic vascularization. Tissue Eng Part A 2015;21:1680-1694. 
30 Arkudas A, Lipp A, Buehrer G, et al: Pedicled transplantation of axially vascularized bone constructs in a critical size femoral defect. Tissue Eng Part A 2018;24:479-492.

31 Eweida AM, Nabawi AS, Abouarab M, et al: Enhancing mandibular bone regeneration and perfusion via axial vascularization of scaffolds. Clin Oral Investig 2014;18:1671-1678.

32 Boos AM, Loew JS, Weigand A, et al: Engineering axially vascularized bone in the sheep arteriovenous-loop model. J Tissue Eng Regen Med 2013; 7:654-664.

33 Arkudas A, Beier JP, Pryymachuk G, et al: Automatic quantitative micro-computed tomography evaluation of angiogenesis in an axially vascularized tissue-engineered bone construct. Tissue Eng Part C Methods 2010;16: 1503-1514.

34 Buehrer G, Balzer A, Arnold I, et al: Combination of BMP2 and MSCs significantly increases bone formation in the rat arterio-venous loop model. Tissue Eng Part A 2015;21:96-105.

$35 \mathrm{Wu}$ X, Wang Q, Kang N, et al: The effects of different vascular carrier patterns on the angiogenesis and osteogenesis of BMSC-TCP-based tissue-engineered bone in beagle dogs. J Tissue Eng Regen Med 2017;11:542552.

36 Dong QS, Shang HT, Wu W, et al: Prefabrication of axial vascularized tissue engineering coral bone by an arteriovenous loop: a better model. Mater Sci Eng C Mater Biol Appl 2012;32:1536-1541.

37 Beier JP, Hess A, Loew J, et al: De novo generation of an axially vascularized processed bovine cancellous-bone substitute in the sheep arteriovenous-loop model. Eur Surg Res 2011;46:148-155.

38 Eweida A, Schulte M, Frisch O, Kneser U and Harhaus L: The impact of various scaffold components on vascularized bone constructs. J Craniomaxillofac Surg 2017;45:881-890.

39 Kim HY, Lee JH, Lee HAR, et al: Sustained release of BMP-2 from porous particles with leaf-stacked structure for bone regeneration. ACS Appl Mater Interfaces 2018;10:21091-21102.

40 Arkudas A, Beier JP, Heidner K, et al: Axial prevascularization of porous matrices using an arteriovenous loop promotes survival and differentiation of transplanted autologous osteoblasts. Tissue Eng 2007;13:15491560.

41 Messina A, Bortolotto SK, Cassell OC, Kelly J, Abberton KM, Morrison WA: Generation of a vascularized organoid using skeletal muscle as the inductive source. FASEB J 2005;19:1570-1572.

42 Bitto FF, Klumpp D, Lange C, Boos AM, Arkudas A, Bleiziffer O, Horch RE, Kneser U, Beier JP: Myogenic differentiation of mesenchymal stem cells in a newly developed neurotised AV-loop model. Biomed Res Int 2013; 2013:935046.

43 Witt R, Weigand A, Boos AM, Cai A, Dippold D, Boccaccini AR, Schubert DW, Hardt M, Lange C, Arkudas A, Horch RE, Beier JP: Mesenchymal stem cells and myoblast differentiation under HGF and IGF-1 stimulation for 3D skeletal muscle tissue engineering. BMC Cell Biol 2017;18:15.

44 Dippold D, Cai A, Hardt M, Boccaccini AR, Horch R, Beier JP, Schubert DW: Novel approach towards aligned PCL-collagen nanofibrous constructs from a benign solvent system. Mater Sci Eng C Mater Biol Appl 2017;72: 278-283.

45 Tee R, Morrison WA, Dusting GJ, et al: Transplantation of engineered cardiac muscle flaps in syngeneic rats. Tissue Eng Part A 2012;18:1992-1999.

46 Tee R, Morrison WA, Dilley RJ: A novel microsurgical rodent model for the transplantation of engineered cardiac muscle flap. Microsurgery 2018;38:544-552.

47 Schmidt VJ, Wietbrock JO, Leibig N, et al: Collagen-elastin and collagen-glycosaminoglycan scaffolds promote distinct patterns of matrix maturation and axial vascularization in arteriovenous loop-based soft tissue flaps. Ann Plast Surg 2017;79:92-100.

48 Brown DL, Meagher PJ, Knight KR, et al: Survival and function of transplanted islet cells on an in vivo, vascularized tissue engineering platform in the rat: a pilot study 1. Cell Transplant 2006;15:319-324.

49 Fiegel HC, Pryymachuk G, Rath S, et al: Foetal hepatocyte transplantation in a vascularized AV-Loop transplantation model in the rat. J Cell Mol Med 2010;14:267-274.

50 Kanapathy M, Patel NM, Kalaskar DM, Mosahebi A, Mehrara BJ, Seifalian AM: Tissue-engineered lymphatic graft for the treatment of lymphedema. J Surg Res 2014;192:544-554.

51 Schaupper M, Jeltsch M, Rohringer S, Redl H, Holnthoner W: Lymphatic vessels in regenerative medicine and tissue engineering. Tissue Eng Part B Rev 2016;22:395-407.

52 Robering JW, Weigand A, Pfuhlmann R, Horch RE, Beier JP, Boos AM: Mesenchymal stem cells promote lymphangiogenic properties of lymphatic endothelial cells. J Cell Mol Med 2018;22:3740-3750.

53 Eweida A, Fathi I, Eltawila AM, et al: Pattern of bone generation after irradiation in vascularized tissue engineered constructs. J Reconstr Microsurg 2018;34:130-137.

54 Beier JP, Horch RE, Arkudas A, et al: De novo generation of axially vascularized tissue in a large animal model. Microsurgery 2009;29:42-51.

55 Schmidt VJ, Wietbrock JO, Leibig N, et al: Haemodynamically stimulated and in vivo generated axially vascularized soft-tissue free flaps for closure of complex defects: evaluation in a small animal model. J Tissue Eng Regen Med 2018;12:622-632.

56 Horch RE, Beier JP, Kneser U, Arkudas A: Successful human long-term application of in situ bone tissue engineering. J Cell Mol Med 2014;18:1478-1485.

57 Zhan W, Marre D, Mitchell GM, Morrison WA, Lim SY: Tissue engineering by intrinsic vascularization in an in vivo tissue engineering chamber. J Vis Exp 2016;111:e54099. 
58 Polykandriotis E, Drakotos D, Arkudas A, et al: Factors influencing successful outcome in the arteriovenous loop model: a retrospective study of 612 loop operations. J Reconstr Microsurg 2011;27:11-18.

59 Weigand A, Boos AM, Ringwald J, et al: New aspects on efficient anticoagulation and antiplatelet strategies in sheep. BMC Vet Res 2013;9:192.

60 Tanaka Y, Sung KC, Tsutsumi A, Ohba S, Ueda K, Morrison WA: Tissue engineering skin flaps: which vascular carrier, arteriovenous shunt loop or arteriovenous bundle, has more potential for angiogenesis and tissue generation? Plast Reconstr Surg 2003;112:1636-1644.

61 Polykandriotis E, Arkudas A, Euler S, Beier JP, Horch RE, Kneser U: Prevascularisation strategies in tissue engineering (in German). Handchir Mikrochir Plast Chir 2006;38:217-223.

62 Polykandriotis E, Schmidt VJ, Kneser U, Jianming S, Boccaccini AR, Horch RE: Bioreactors in regenerative medicine-from a technical device to a reconstructive alternative? (in German). Handchir Mikrochir Plast Chir 2012;44:198-203.

63 Schmidt VJ, Covi JM, Koepple C, et al: Flow induced microvascular network formation of therapeutic relevant arteriovenous (AV) loop-based constructs in response to ionizing radiation. Med Sci Monit 2017;23:834-842.

64 Horch RE, Weigand A, Wajant H, Groll J, Boccaccini AR, Arkudas A: Biofabrication: new approaches for tissue regeneration (in German). Handchir Mikrochir Plast Chir 2018;50:93-100.

65 Hutmacher DW, Horch RE, Loessner D, et al: Translating tissue engineering technology platforms into cancer research. J Cell Mol Med 2009;13:1417-1427.

66 Weigand A, Tasbihi K, Strissel PL, Strick R, Horch RE, Boos AM: Development of an innovative cell isolation method for the investigation of breast cancer pathogenesis and angiogenesis for experimental in vitro and in vivo assays (in German). Handchir Mikrochir Plast Chir 2017;49:111-122. 